\title{
Robust optimisation of cardiovascular stents: a comparison of methods
}

\author{
R A Bates and M A Atherton
}

\begin{abstract}
Modern engineering design contains both creative and analytic components. This paper discusses the design process and illustrates links between design optimisation and conceptual design through the re-design of a cardiovascular stent. A comparison is presented of two methods for design improvement: Genetic Algorithms (GA) and model-based Robust Engineering Design (RED). Computational Fluid Dynamics (CFD) models are used to generate measurements of the quality of competing designs based on the concept of dissipated power. Alternative performance measures are also discussed. Environmental noise is introduced into the analysis and consideration is given to the treatment of discrete and continuous design parameters. Improved designs are identified using both methods and verified with further CFD analyses, and the benefits of each method are discussed.
\end{abstract}

\section{Introduction}

The field of engineering design and optimisation has been revolutionised by the continual increase in computing power. Methods such as Computational Fluid Dynamics (CFD) and Finite Element Analysis (FEA) can be used to analyse the performance of new designs and allow several designs to be compared so that the best ones are finally chosen. This design optimisation process is the subject of much study, and the boundaries between creative design and design analysis are sometimes not clear. For example, evolutionary computing methods seek to automate the process of searching for solutions, given a set of design rules. However, such methods always require a mathematical framework to function, and therefore in some way, the space of all possible solutions is defined at the start, precluding the selection of any revolutionary design that may not be included in the defined space.

This paper focusses on re-design, or design improvement, by comparing two methods, Genetic Algorithms (GA) and model-based Robust Engineering Design (RED) applied to the problem of how to design cardiovascular stents. Stents are metallic cage like structures that are inserted into an artery that has become fully or partially blocked by calcified plaque (stenosis). Their function is to maintain the flow of blood, with the constraints that the stent must be able to contract and expand to enable insertion, and that it must have a minimal effect on the flow of blood through the artery. In addition, the stent needs to work for a variety of shapes and sizes of artery, both of which can vary significantly across gender and race. 


\subsection{Design: art vs. science}

Engineering design can be viewed as both an art and a science [1,2]. By this it is meant that there are some aspects of the design process that rely on learning by doing (art), and others concerned with developing method or procedure (science). Comparing scientific method with the procedure of engineering design draws out two important points. First, the impetus for science is the thirst for knowledge and the impetus for design stems from the needs of society [2]. Second, in science, problems are explored for their underlying rules, i.e. a problem-focused approach, whereas in design the quest is to suggest possible solutions, i.e. a solution-based approach [3].

In relation to cardiovascular stents, the overall process could be interpreted as 'scientific' activity when concerned with understanding haemodynamics and 'design' activity when concerned with employing this understanding in seeking to reduce rates of restenosis, see Table 1.

\begin{tabular}{l|ll} 
& Impetus & Objective \\
\hline Science & seek knowledge & understand haemodynamics \\
Design & satisfy demand & reduce restenosis
\end{tabular}

Table 1: Role of science and design in cardiovascular stent research

\subsection{Types of design problem}

Types of design problems may be described as original design, redesign or routine design [4]. Original design is concerned with radical new working principles or innovative features. The focus of redesign is on modifying an existing working principle or changing the arrangement of important features. Routine design is typified by the detail changes required to produce a new size within a common range of components. Thus the types of design problems are distinguished by the degree of creative effort deployed on the working principle as opposed to detailed refinements. In the context of this study stents are considered to be a redesign problem. This is by virtue of aiming to reduce restenosis through changes to the arrangement of important features of the stent rather than relying on a radically new working principle. Table 2 provides a summary.

\begin{tabular}{l|l} 
Original design & $\begin{array}{l}\text { Working principle = expanding structure that dilates the } \\
\text { stenosis and allows blood to flow. }\end{array}$ \\
\hline Redesign & $\begin{array}{l}\text { Arrangement of structural elements in relation to } \\
\text { interaction with artery wall and blood flow characteristics. }\end{array}$ \\
\hline Routine design & Geometry values for different sizes of stent.
\end{tabular}

Table 2: The stent design problem

Stents have developed over the past fifteen years from simple designs woven out of fine wire [5] to more elaborate designs laser-cut from thin cylinders. The diversity of current expanding stent designs is evident from considering some typical stent designs, shown in Figure 1. 

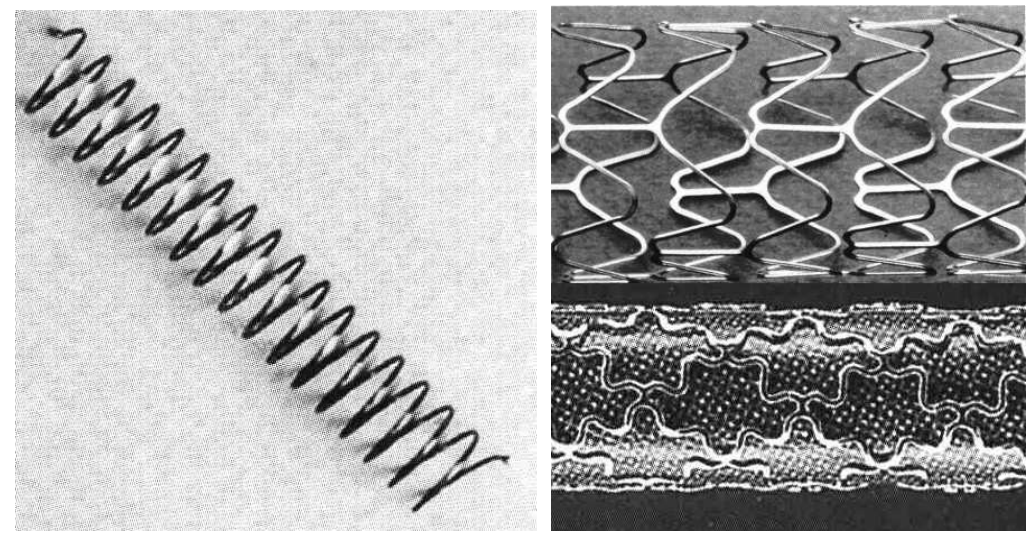

Figure 1: Typical stent designs. Left: early design. Right: two modern designs.

\section{The design of stents}

Stent designs were initially developed to solve the problem of structural support. However, the importance of designing for blood flow characteristics is now recognized [6] as the overriding concern. This is because flow is believed to be a key contributing factor in the re-blockage (restenosis) of a stented artery. Therefore stent pattern features and strut details are now designed with fluid flow in mind.

The result is that stent design has developed away from the relatively dense simple helical-type designs to more open and sophisticated forms made from thinner material that are less disruptive to the blood flow. The role of Wall Shear Stress (WSS) in restenosis has received much attention [7] and to this end, Computational Fluid Dynamics (CFD) has emerged as a vital design tool. It is interesting that WSS cannot be measured in vivo as yet, which restricts direct validation of results obtained from computer simulations using CFD methods. Another constraint is due to the physical size of the stent itself. The ratio of overall size to important detail is large for a stent, typically about 30, and this means that the CFD stent model needs to be discretized into a fine mesh to achieve accurate calculations, and this in turn requires a large amount of computation power. Therefore full stent models can be unsatisfactory, as they do not fully resolve the recirculation regions around stent struts and this limits the degree of confidence in finding an optimum design.

\subsection{Design search}

In an automatic search for a good design, questions that a scientist might ask are:

1. What is the space of all possible stent designs?

2. How can we set up a mathematical framework to search for an improved stent design?

The underlying rules uncovered in science are unique to certain aspects of the problem whereas in design there may be many solutions for satisfying a stated need. Thus the engineering design process is generally concerned with exploring design situations, exploring the problem structure, searching for ideas and evaluating solutions [8]. The rational aspects of such are more commonly regarded as design methods or procedures as they encourage a systematic approach. It is convenient to picture the engineering design 


\begin{tabular}{l|l} 
Characteristic & Typical values \\
\hline Pattern description & $\begin{array}{l}\text { Rings with links at 120deg, diamond, sine wave, } \\
\text { zig-zag rings with spine }\end{array}$ \\
Strut thickness & $0.05 \mathrm{~mm}$ to $0.15 \mathrm{~mm}$ \\
Degree of cross-struts (> 80deg) & $7 \%$ to $77 \%$ \\
Max. axial space between struts & $0.7 \mathrm{~mm}$ to $3 \mathrm{~mm}$ \\
Max. circumferential spacing & $0.7 \mathrm{~mm}$ to $4.7 \mathrm{~mm}$
\end{tabular}

Table 3: Typical values of stent design features

process as a search in an imaginary space in which the goal is to find the solution(s) that satisfies specified needs. The search approach adopted depends to a large extent on the type of design problem. Conventionally, a random search is employed in original design, whilst redesign is accomplished in a heuristic fashion through improving understanding of the problem and in turn finding a better solution. This approach can however be costly, and can also result in sub-optimal solutions. In contrast, routine design as described in Table 1 requires virtually no search activity.

\subsection{Typical stent designs}

Early stent designs were woven from fine wire [5] but today more elaborate designs are used, commonly laser-cut from a thin cylinder or sheet. Examples of both early and modern designs are shown in Figure 1.

Thus the numbers of possible contemporary design configurations is very large and coverage of the associated design space requires efficient design procedures to search for the best design solutions. Table 3 summarises some of the basic features of stents from a comparison of several products [9]. The number of design factors that might affect these features is estimated to be in the region of 4 to 10 .

Table 4 shows typical values for the structural characteristics of stents as summarised from data available in the literature [10].

\begin{tabular}{l|l} 
Characteristic & Typical values \\
\hline (a) Radial force & Good to excellent \\
(b) Longitudinal flexibility & low to excellent \\
(c) Contact area & $12 \%$ to $20 \%$ \\
(d) Shortening on expansion & 0 to $9.5 \%$ \\
(e) Recoil & $1 \%$ to $4.8 \%$
\end{tabular}

Table 4: Typical values of stent structural characteristics

\subsection{Parameterization of stents}

Stent design looks relatively simple, but the degrees of freedom of the patterns mean there are thousands of potential solutions. These shapes can also be rather elaborate and difficult to describe in simple terms. In order to compare different design methods, we now present a small design study concentrating on the five main features listed below in Table 5. The first three of these parameters are: (i) strut thickness, ranging from 0.08 to $0.10 \mathrm{~mm}$, (ii) cross-section, which is represented as a ratio of thickness to width, and (iii) 
asymmetry or skew. The effects that changing these parameters has on the stent design is shown in Figure 2.
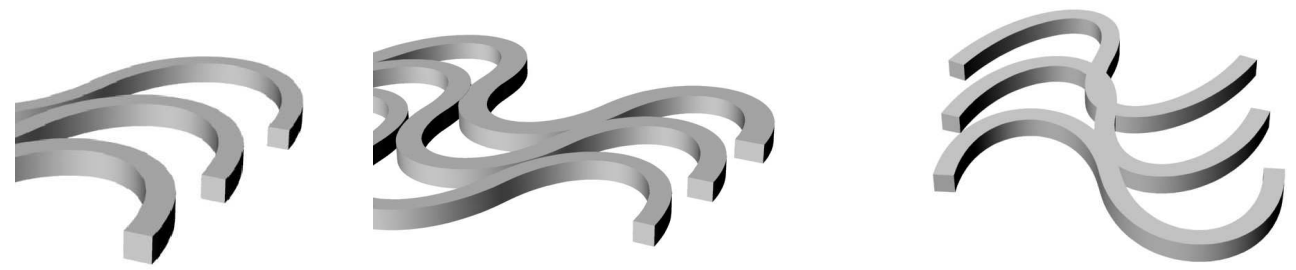

Figure 2: Variation of strut thickness (left), width (centre) and skew (right).

A skew value of 0.5 represents a symmetrical pattern and a value of 0.9 produces asymmetry through bringing the two peaks of the pattern closer together. Repeating Pattern determines whether the next stent segment is merely a copy or a mirror image of the existing segment. If a segment is copied then an artificial link must be added to join the two segments together. A mirror operation does not necessarily need any linking elements because segments are joined naturally, see Figure 3.
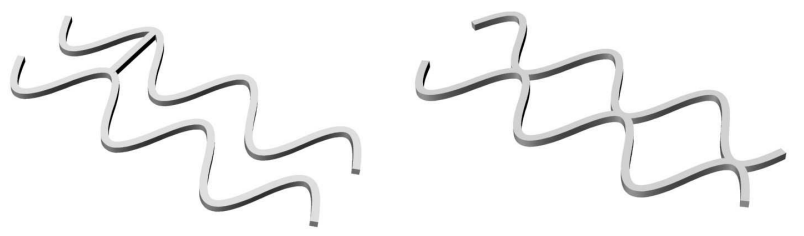

Figure 3: Copy and mirror stent designs

Shape Order, illustrated in Figure 4, defines the degree of curvature of the segments. In this particular case '1st order' produces a sharp definition and '2nd order' produces a smoother definition.
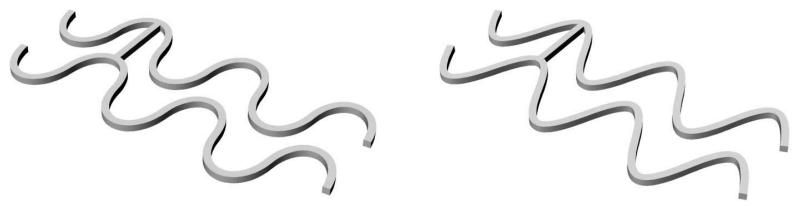

Figure 4: Variation in curvature of stent

\begin{tabular}{l|l} 
Name & Range \\
\hline Strut Thickness d (mm) & $0.08-0.10$ \\
Strut Thickness:Width ratio & $1: 1-1: 1.5$ \\
Pattern Skew & $0.5-0.9$ \\
Repeating Pattern & Copy/Mirror \\
Shape Order & 1 st and 2nd
\end{tabular}

Table 5: Stent pattern description 
The five variables, or factors described above define the design space that will be searched in order to find the stent design that minimises wall sheer stress (WSS). This measure is associated with restenosis when its value is too high or too low. However in order to estimate the quality of the design, it is more convenient to choose dissipated power as a surrogate for WSS, as it provides a scalar quantity rather than WSS which must be summarised by, for example, averaging over the whole stent surface. Indeed, dissipated power was inspired by Murray's Law [11], which states that arterial trees are observed to branch into smaller arteries according to the minimization of energy losses rather than following a constant area law.

For computational purposes, it is convenient to work with a partial model of a stent rather than a full 3D model, which could be too costly in terms of computation time. Even with this simplification it is not practical to exhaustively test every possible design. Instead a search strategy needs to be employed to find the optimal solution.

\section{Methods for redesign}

\subsection{Application of Genetic Algorithms}

Genetic Algorithms (GA) are a class of global search strategies that attempt to find optimal design solutions by borrowing from theories of evolutionary adaptation. The framework of GA's is to parameterize the design problem into discrete factors, that is factors that take only a fixed number of values. Every possible design solution can then be encoded in a binary string, analogous to a genetic chromosome. The string can then be subjected to the equivalent genetic operations of mutation, crossover and so on, in the search for an optimal solution.

The parameterization of the stent, described in the previous section, is used to define a GA search method to find an improved stent design. Two alleles are used for both 'ratio' and 'thickness' in encoding the GA chromosome. As these factors have only three values, and the alleles can represent $2^{2}=4$ values, a dummy level is necessary, and the fourth value in the allele combination is made equal to the third value.

The full parameter encoding for the stent chromosome is shown in Table 6 below. The total length of the chromosome is 9 in representing the 5 factors.

\begin{tabular}{|c|c|c|c|c|c|c|c|c|}
\multicolumn{2}{|c|}{ ratio } & \multicolumn{2}{|c|}{ thickness } & \multicolumn{3}{|c|}{ skew } & copy & shape \\
\hline 0 & 1 & 1 & 0 & 0 & 1 & 1 & 1 & 0 \\
\hline
\end{tabular}

Table 6: Parameter encoding

The above encoding presents us with $2^{9}=512$ possible combinations of design factors. However, some combinations are repeated because of the dummy level, and so there are 288 unique cases. Because an analysis of each case can take at least an hour, only a subset of all the possible designs can be evaluated, and the GA evaluates samples from the 288 possible designs in order to optimize the stent.

Brief details of the GA search parameters are now given, a full description is given in a recent paper [12]. Due to the relatively short chromosome length and the high cost of calculation, the population size was chosen to be 10 . Crossover probability was set at 0.75 and mutation probability at 0.02 to avoid local extreme convergence. The total number of generations passed to convergence was 11. During the calculation process 20 mutations 
and 40 crossovers were produced. The total number of unique stent shapes tested was 27 , which is about $10 \%$ of the whole (discrete) design space.

\subsubsection{GA robustness measure}

The aim of the search is to find a stent design that minimizes the effect of the stent on the flow of blood. This effect is approximated by measuring dissipated power (diss) and minimizing this quantity. In order to incorporate the notion of robustness in the search for an optimal solution, two noise factors were introduced into the study. The first noise factor represents the amount of embedding of the stent in the artery wall. This can vary significantly between patients and clearly affects the flow of blood through the artery. The second noise factor represents the angle of fluid flow. This is important when considering that no artery is totally straight, and the stent may also be placed near a branch in the artery.

For each test, dissipated power (in watts) was measured four times at all two-level combinations (high and low) of each noise factor. The response SSdiss was taken as the sum of squares of these four measurements.

\subsubsection{GA results}

Table 7 shows the optimal design found using the GA approach, which is illustrated in Figure 5 .

\begin{tabular}{l|l} 
Name & Value \\
\hline Strut Thickness d $(\mathrm{mm})$ & 0.08 \\
Strut Thickness:Width ratio & $1: 1$ \\
Pattern Skew & 0.5 \\
Repeating Pattern & Mirror \\
Shape Order & 1 st
\end{tabular}

Table 7: GA results

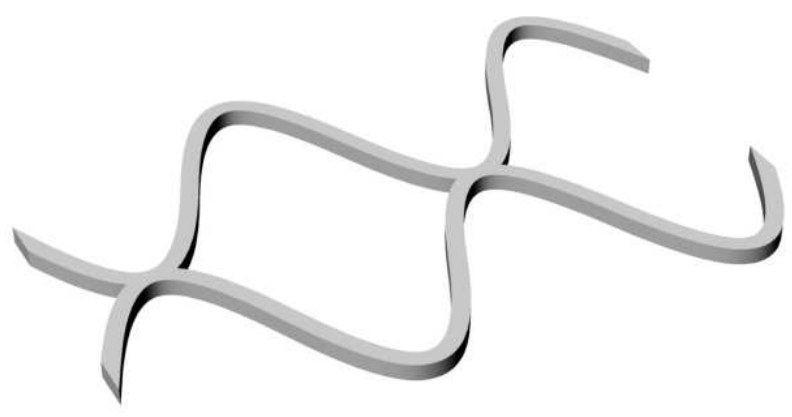

Figure 5: Optimal design found using GA.

The optimal design gave a value of SSdiss $=92.25 \times 10^{-6} \mathrm{~W}^{2}\left(\right.$ Watts $\left.^{2}\right)$. 


\subsection{Application of Statistical Methods}

The GA performs a discretisation of the search space which leads to a finite set of possible solutions. However, the first 3 design factors are actually continuous, not discrete. Instead an alternative approach using design of experiments can be more efficient in finding an optimal design. To try and find a robust design solution, some of the real-life variation that a stent will encounter is included in the analysis.

The model-based Robust Engineering Design (RED) approach adopted here is summarized as follows:

1. experiment: conduct a set of simulations at chosen input factor values (both design and noise factors),

2. emulate: fit a statistical model, an emulator, to the experimental data, and

3. optimize: perform global numerical optimization using the emulator to construct the objective function.

The emulator and optimizer stages require the design and noise factors to be continuous, so the two discrete design factors need to be treated individually. Initial studies confirmed the best discrete factor combination to be a first-order strut shape with mirrored symmetry, so rather than perform unnecessary trials, this setting was fixed and a single experiment of 12 trials was performed on the three remaining design factors and the two noise factors. Table 8 shows the results of experimentation.

\begin{tabular}{|c|c|c|c|c|c|}
\hline \multirow[b]{2}{*}{ run no. } & \multicolumn{5}{|c|}{ continuous factor setting } \\
\hline & skew & thickness (mm) & width ratio & embedding (\%) & inlet angle (deg) \\
\hline 1 & 0.65 & 0.087 & 1.00 & 36.36 & 60.00 \\
\hline 2 & 0.57 & 0.089 & 3.55 & 07.27 & 00.00 \\
\hline 3 & 0.79 & 0.093 & 1.73 & 21.82 & 10.91 \\
\hline 4 & 0.72 & 0.095 & 2.45 & 50.91 & 54.55 \\
\hline 5 & 0.54 & 0.100 & 2.09 & 43.64 & 32.73 \\
\hline 6 & 0.90 & 0.098 & 3.91 & 58.18 & 27.27 \\
\hline 7 & 0.68 & 0.091 & 4.64 & 14.55 & 43.64 \\
\hline 8 & 0.86 & 0.096 & 2.82 & 65.45 & 21.82 \\
\hline 9 & 0.50 & 0.080 & 1.36 & 0.000 & 16.36 \\
\hline 10 & 0.83 & 0.082 & 4.27 & 72.73 & 5.45 \\
\hline 11 & 0.76 & 0.084 & 5.00 & 29.09 & 38.18 \\
\hline 12 & 0.61 & 0.086 & 3.18 & 80.00 & 49.09 \\
\hline
\end{tabular}

Table 8: RED experimental design plan

For discrete problem $D_{3}$, a DACE emulator [13] is fitted that can then be used to predict the response for any set of values for the 3 continuous factors and 2 noise factors. The emulator acts as a fast approximation to the CFD code and can be used to examine the effect that each factor has on the response. Figure 6 shows a plot, called the main effects plot, describing the individual relationships between each factor and the response. 

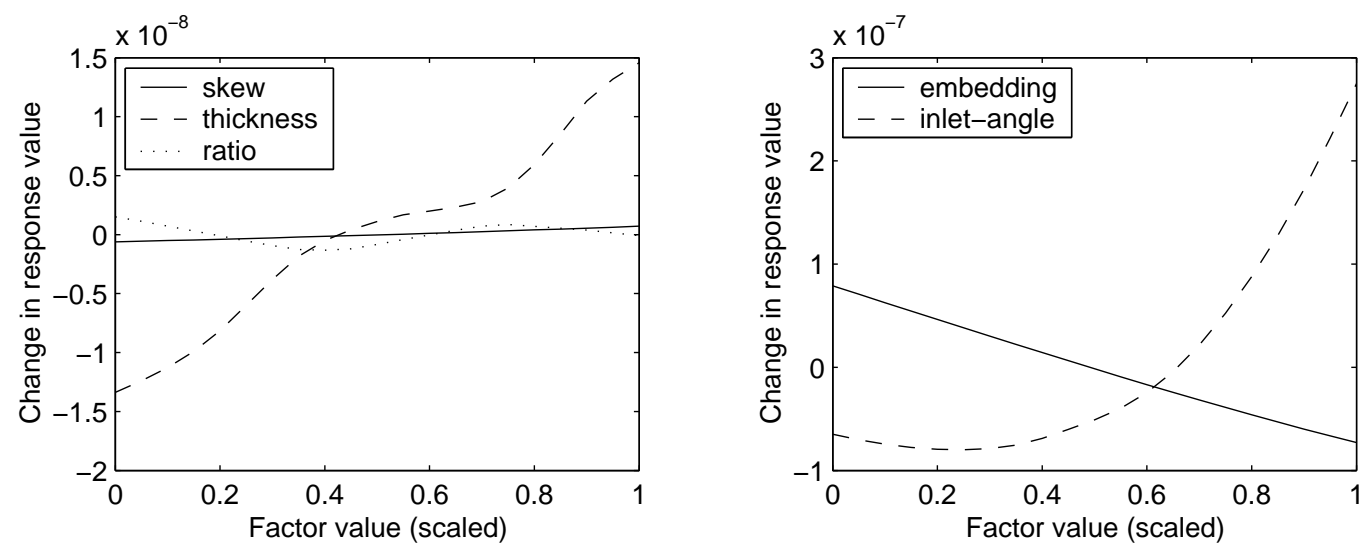

Figure 6: Emulator main effects plot (response values in $\mathrm{W}^{2}$ ).

One can see that the inlet angle noise factor and the strut thickness design factor both have a nonlinear effect on the response, which is of interest when considering design solutions that are robust to noise. The emulator is combined with a numerical global optimizer to determine the values for the 3 continuous design factors that yield the lowest value for the sum of squares of the response at the 4 noise factor settings described in the previous section on the GA study $\left(S S d i s s_{1}, \ldots, S S d i s s_{4}\right)$. This measure was chosen so the results of the two studies could be compared. The results of the optimization process are shown in Table 9 .

\begin{tabular}{cccccc}
$\begin{array}{c}\text { width } \\
\text { ratio }\end{array}$ & $\begin{array}{c}\text { thickness } \\
(\mathrm{mm})\end{array}$ & skew & $\begin{array}{c}\text { predicted } \\
\text { SSdiss }\left(\mathrm{W}^{2}\right)\end{array}$ & $\begin{array}{c}\text { computed SSdiss } \\
\text { using CFD }\left(\mathrm{W}^{2}\right)\end{array}$ \\
\hline$D_{3}$ & 1.5 & 0.08 & 0.518 & $89.70 \times 10^{-6}$ & $91.07 \times 10^{-6}$
\end{tabular}

Table 9: Predicted results using emulator

Table 9 shows that the optimal solution has an estimated response of $\widehat{S \text { Sdiss }}=89.70 \times$ $10^{-6} \mathrm{~W}^{2}$ using the emulator model. Running the CFD simulator at this setting gives SSdiss $=91.08 \times 10^{-6} \mathrm{~W}^{2}$ which is in close agreement.

\section{Comparison of methods and conclusions}

One can immediately see that the two methods described treat noise and robustness differently. The use of two levels for noise (high and low) for the GA study immediately assumes that noise affects design in a linear fashion, whereas the RED study shows that inlet angle has a nonlinear effect. Measuring noise at only high and low levels will therefore not accurately reflect the true impact of noise on the response. The discretization of the design space by the GA method means that discrete variables such as competing architectures are considered naturally. However, considering the remaining continuous design factors as discrete restricts the search for an improved design and does not allow any analysis to be made on how the design factors are affecting the response. The RED method on the other hand treats the continuous factors as continuous and searches a larger space of designs as a result. The penalty for this is that each discrete case must be considered separately. The RED method does however provide insight into the design problem through analysis, and this may aid the designer in understanding the design 
problem and help in finding improved design solutions. Both the GA and RED searches found improved designs, the RED design giving slightly better results. It is also interesting to note that the GA search required $27 \times 4=108 \mathrm{CFD}$ simulations, whereas the RED search required a maximum of $12 \times 4=48 \mathrm{CFD}$ simulations.

In this medical engineering example, the stent device utilizes technology with a static function, i.e. a single pattern that best accommodates the noise distribution experienced between patients. Ignoring other design objectives, a dynamic solution, if it were possible, would adopt whatever shape is necessary to minimize disruption to the flow. Therefore it is interesting to observe that contemporary stent designs commonly use symmetric patterns - why? It could be that noise is not considered at the design stage, which corresponds to the assumption that the blood flow is purely axial.

Another point for consideration is the choice of performance measure or objective function used to drive stent design development. For arterial blood flows there are uncertainties in making a link between wall shear stress (WSS) and restenosis, and between WSS and dissipated power as a performance measure. Following Murray's law, we have considered dissipated power as a performance measure inspired by nature as a logical option. Murray also showed that the minimization of flow resistance in a complex manifold of vessels is achieved when the wall shear stress on all walls is equal. Hence this is a justification for our choice of dissipated power as a surrogate for WSS. As we might expect, dissipated power is essentially a bulk flow measure and it is therefore sensitive to the inflow angle. Whereas WSS is a local measure of work expended against the wall and the CFD package used only produces its scalar quantity, which could account for slight differences in the designs that have emerged from additional studies performed using the two performance measures.

A large design space is produced by the few design factors considered, some of which were limited by the constraints of producing a CFD model that will mesh satisfactorily. However this is not a search of technology options, rather it is a parameter search for improvement of an existing working principle, in other words adaptation. Neither GA nor RED searches can create new technologies. The GA search converged effectively within a few generations but choosing appropriate values for mutation and crossover was an additional uncertainty in configuring the search.

These studies both show the challenges involved in trying to automate the redesign process and incorporating noise into the design process more generally. The search method chosen will normally depend on the amount of time and resources available to the designer. In this regard, the RED method is more efficient as it required less design evaluations than the GA approach, and achieved a better result. It is important to note that the way a design is parameterized, and the way competing designs are judged is critical in determining the success of any strategy for design improvement. As an illustration of this, the use of dissipated power as a measure for improving the design seems to work well, and is consistent with nature. If sources of noise are not taken into account, then an improved design will not necessarily be robust to them and may fail as a result.

\section{References}

[1] G Pahl and W Beitz. Engineering design - a systematic approach. Springer-Verlag, 1988. Wallace K, ed.

[2] G Dieter. Engineering design. McGraw-Hill, 1983. 
[3] B R Lawson. Cognitive strategies in architectural design. In N Cross, editor, Developments in Design Methodology, pages 209-220. Wiley, 1984.

[4] N F O Evbuomwan, S Sivaloganathan, and A Jebb. A survey of design philosophies, models, methods and systems. Proc. Inst. Mech. Engrs., 210:301-320, 1996.

[5] U Sigwart, J Puel, V Mirkovitch, F Joffre, and L Kappenberger. Intravascular stents to prevent occlusion and restenosis after transluminal angioplasty. New England J. of Medicine, 316(12):701-706, 1987.

[6] Y Yoshitomi et al. Does stent design affect probability of restenosis? a randomized trial comparing multilink stents with gfx stents. American Heart Journal, 142(3):445451, 2001.

[7] A H Goodhall. Role of shear stress and turbulence on platelets in blood conduits and on endothelial cells in arterial conduits. In U Sigwart, editor, Endoluminal Stenting, pages 65-78. WB Saunders, London, 1996.

[8] J Jones. Design methods. Wiley, 1981.

[9] M A Atherton, D J Doorly, and M W Collins. Applying design procedures to cfd models for improved stent designs. Proc. Of Cardiovascular Fluid Dynamics, IMechE, 1999.

[10] P W Serruys and M J B Kutryk. Handbook of coronary stents. Martin Dunitz, 2nd edition, 1998.

[11] C D Murray. The physiological principle of minimum work. i. the vascular system and the cost of blood volume. Proc. Nat. Acad. Sci. USA, 12:207-214, 1926.

[12] K Tesch, M A Atherton, and M W Collins. Genetic algorithm search for stent design improvements. In I C Parmee, editor, Adaptive Computing in Design and Manufacture $V$, pages 99-107. Springer, 2002.

[13] J Sacks, W J Welch, T J Mitchell, and H P Wynn. Design and analysis of computer experiments. Statistical Science, 4:409-435, November 1989. 